\title{
GESTÃO DA CADEIA DE SUPRIMENTOS INTEGRADA À TECNOLOGIA DA INFORMAÇÃO - ABORDAGEM QUALITATIVA EM UM GRUPO EMPRESARIAL BRASILEIRO
}

Rafael Pontes Cantal ${ }^{1}$ rafaelcantal@hotmail.com

PROF. M. Sc. Rickardo Léo Ramos Gomes ${ }^{2}$ rickardolrg@yahoo.com.br https://orcid.org/0000-0001-6101-9571

Para citar este artículo puede utilizar el siguiente formato:

Rafael Pontes Cantal y Rickardo Léo Ramos Gomes: "Gestão da cadeia de suprimentos integrada à tecnologia da informação - abordagem qualitativa em um grupo empresarial brasileiro", Revista Observatorio de la Economía Latinomericana (Vol 19, № 5, pp. 60-72, julio-septiembre 2021). En línea:

https://doi.org/10.51896/oel/XXHE4003

\section{RESUMO}

A moderna gestão da cadeia de suprimentos teve grande impulso com o advento da tecnologia da informação e com o desenvolvimento dos sistemas de informação, tornando-se mais bem planejada e integrada, possibilitando um melhor controle e gestão dos processos. Nesta pesquisa demonstrase o quanto esta integração é importante para evolução da Supply Chain Management (SCM). O objetivo geral desse trabalho foi demonstrar a importância da tecnologia da informação e sistema da informação na SCM, apresentando o modelo que foi implantado para a melhoria da integração entre as empresas. Os objetivos específicos ficaram assim sequenciados: descrever a cadeia de suprimentos; demonstrar a importância da tecnologia da informação e sistemas de informação; abordar a tecnologia da informação e sistemas de informação na cadeia de suprimentos; relatar os impactos da automatização da cadeia de suprimentos e discorrer sobre a aplicação da tecnologia da

\footnotetext{
${ }^{1}$ Graduado em Ciência da Computação pela Universidade de Fortaleza - UNIFOR; Pós-graduando do curso MBA em Gestão de Suprimentos pelo Instituto Euvaldo Lodi (IEL).

2 Prof. da Disc. de Met. do Trabalho Científico (Orientador) - Inst. Euvaldo Lodi; C. U. UniAteneu; C. U. Farias Brito; M. Sc. em Fitotecnia pela Universidade Federal do Ceará (UFC); Spec. em Met. do Ens. de Ciências pela UECe; Grad. em Agronomia pela UFC; Licenciado na Área de Ciências da Natureza, Matemática e suas Tecnologias pela UVA; Aperf. em Líderes de Aprendizagem pela Universidade de Harvard; Aperf. em Gestão de Riscos em Projetos pelo BID; Aperf. em Met. do Trabalho Científico pela FIOCRUZ. Curso Aperf. Rastreamento do Contato da COVID-19 pela Johns Hopkins University (JHBSPH); Consultor Internacional do BIRD para Laboratórios Científicos. Conveniado com a ABNT (Comitês 093 e 130). Fundador da RLRG Consultoria Científica.
} 
informação na cadeia de suprimentos de um grupo empresarial brasileiro. O presente trabalho seguiu uma abordagem qualitativa com caráter exploratório e descritivo e a metodologia empregada foi a da pesquisa bibliográfica, que utilizou a análise de referências teóricas publicadas em livros, artigos científicos e documentos. Ao final desse trabalho foi possível avaliar que a Tecnologia da Informação vem atuando como forte aliada na evolução da SCM na busca da redução de custos e no enfrentamento da competitividade.

Descritores - Tesouro da UNESCO: TG: Sistema de informação; TE: Sistema de Informação Integrada; Centro de Dados; Serviços de Informação.

Palavras-chave: Cadeia de suprimentos. Tecnologia da informação. Sistemas de informação.

\section{GESTIÓN DE LA CADENA DE SUMINISTRO INTEGRADA A LA TECNOLOGÍA DE LA INFORMACIÓN - ENFOQUE CUALITATIVO EN UN GRUPO EMPRESARIAL BRASILEÑO}

\section{RESUMEN}

La gestión moderna de la cadena de suministro tuvo un gran impulso con el advenimiento de la tecnología de la información y con el desarrollo de los sistemas de información, volviéndose más bien planificados e integrados, permitiendo un mejor control y gestión de los procesos. Esta investigación demuestra la importancia de esta integración para la evolución de Supply Chain Management (SCM). El objetivo general de este trabajo fue demostrar la importancia de las tecnologías de la información y los sistemas de información en SCM, presentando el modelo que se implementó para mejorar la integración entre empresas. Los objetivos específicos se secuenciaron de la siguiente manera: describir la cadena de suministro; demostrar la importancia de la tecnología de la información y los sistemas de información; abordar la tecnología de la información y los sistemas de información en la cadena de suministro; informar los impactos de la automatización de la cadena de suministro y discutir la aplicación de la tecnología de la información en la cadena de suministro de un grupo empresarial brasileño. El presente trabajo siguió un enfoque cualitativo con carácter exploratorio y descriptivo y la metodología utilizada fue la de investigación bibliográfica, que utilizó el análisis de referencias teóricas publicadas en libros, artículos científicos y documentos. Al final de este trabajo, se pudo evaluar que las Tecnologías de la Información han venido actuando como un fuerte aliado en la evolución de la SCM en la búsqueda de la reducción de costos y de cara a la competitividad.

Descriptores - Tesauro de la UNESCO: TG: Sistema de información; TE: Sistema de Información Integrado; Centro de datos; Servicios de información.

Palabras clave: Cadena de suministro. Tecnología de la información. Sistemas de información.

\section{SUPPLY CHAIN MANAGEMENT INTEGRATED TO INFORMATION TECHNOLOGY - QUALITATIVE APPROACH IN A BRAZILIAN BUSINESS GROUP}

\section{ABSTRACT}

The modern management of the supply chain had a great impulse with the advent of information technology and with the development of information systems, becoming more well-planned and integrated, enabling better control and management of processes. This research demonstrates how 
important this integration is for the evolution of Supply Chain Management (SCM). The general objective of this work was to demonstrate the importance of information technology and information system in SCM, presenting the model that was implemented to improve the integration between companies. The specific objectives were sequenced as follows: describe the supply chain; demonstrate the importance of information technology and information systems; address information technology and information systems in the supply chain; report the impacts of supply chain automation and discuss the application of information technology in the supply chain of a Brazilian business group. The present work followed a qualitative approach with an exploratory and descriptive character and the methodology used was that of bibliographical research, which used the analysis of theoretical references published in books, scientific articles and documents. At the end of this work, it was possible to assess that Information Technology has been acting as a strong ally in the evolution of SCM in the search for cost reduction and facing competitiveness.

Descriptors - UNESCO Treasury: TG: Information system; TE: Integrated Information System; Data Center; Information Services.

Keywords: Supply chain. Information Technology. Information systems.

\section{INTRODUÇÃO}

Com um mercado, cada vez mais competitivo, as empresas buscam ferramentas e processos que auxiliem na melhoria de seus processos, possibilitando inovações e aumentando desempenho e competitividade, com foco em redução de custos. Dessa forma, visando integrar as diversas áreas das organizações, a gestão da cadeia de suprimentos vem apresentando-se como uma grande aliada para a obtenção dessas vantagens competitivas. Percebeu-se que a gestão da cadeia de suprimentos seria mais eficiente com a prática dos conceitos de tecnologia da informação e sistemas de informação, onde essa cadeia poderia ser mais bem planejada e integrada, possibilitando o controle e gestão dos processos, desde a contratação dos fornecedores, passando por toda a cadeia, e com o produto chegando ao consumidor final, observando todos os pontos que podem impactar no processo.

Todas essas evoluções supracitadas só seriam possíveis através do uso da tecnologia da informação. Para isso, sistemas integrados de informações tornam-se, cada vez mais, necessários para promover a inovação e a disseminação do conhecimento ao longo da cadeia de abastecimento, de forma a melhorar a qualidade e produtividade, garantindo, assim, a competitividade organizacional de todo o processo. Nos últimos anos a TI (Tecnologia da Informação) e SI (Sistema de Informação) trouxeram avanços significativos para a cadeia global de suprimentos. Grande parte dessa melhoria deve-se a uma comunicação mais rápida, gerando um dinamismo e uma tomada de decisão mais eficaz, afetando toda a cadeia de suprimentos. O gerenciamento da cadeia de suprimentos (SCM Supply Chain Management), é um conceito que propõe processos enxutos, eliminação de desperdícios e redução de estoques (Stéen, 2006). 
Os modelos de SCM utilizados atualmente, não atingem os resultados esperados e, muitas vezes, existe uma grande lacuna entre os benefícios reais e potenciais. Desta forma, torna-se decisivo, para as organizações, a utilização de sistemas de informações e TI eficientes, integrando e suportando toda a cadeia de suprimentos. O uso de um ou vários sistemas pode integrar as empresas que participam da cadeia de abastecimento e repensar as condições que as ajudam a responder aos desafios da vida diária de forma eficiente e duradoura. (Martins, 2011)

Isso também se aplica à realidade competitiva das empresas do mercado brasileiro, visto que elas estão necessitando melhorar seus modelos de gestão e integração para apoiar o crescimento estratégico, facilitando o enfrentamento à competição global. Nesse estudo será apresentado um grupo empresarial, atuante em todo território nacional, abrangendo diversos segmentos de mercado.

O objetivo geral desse trabalho é demonstrar a importância da tecnologia da informação e sistema da informação na Supply Chain Management (SCM), apresentando o modelo que foi implantado para a melhoria da integração entre essas empresas, agilizando os processos e garantindo o controle da cadeia de suprimentos.

Os objetivos específicos ficaram assim sequenciados: descrever a cadeia de suprimentos; demonstrar a importância da tecnologia da informação e sistemas de informação; abordar a tecnologia da informação e sistemas de informação na cadeia de suprimentos; relatar os impactos da automatização da cadeia de suprimentos e discorrer sobre a aplicação da tecnologia da informação na cadeia de suprimentos de um grupo empresarial brasileiro.

O presente trabalho seguiu uma abordagem qualitativa com caráter exploratório e descritivo e a metodologia empregada foi a da pesquisa bibliográfica, que utilizou a análise de referências teóricas publicadas em livros, artigos científicos e documentos, para que fosse possível explicar, com maior aprofundamento, os assuntos contidos nesse trabalho, à luz das contribuições científicas preexistentes.

O artigo ficou estruturado em quatro tópicos. No primeiro elaborou-se a introdução com contextualizações importantes sobre a temática pesquisada. No segundo elaborou-se a fundamentação teórica com múltiplas discussões entre autores que estão relacionados com 0 mesmo assunto aqui abordado. No terceiro foram apresentados os procedimentos metodológicos adotados para elaboração do artigo e o quarto, e último, tópico foi reservado para as considerações finais.

\section{METODOLOGIA}

O presente trabalho seguiu uma abordagem qualitativa com caráter exploratório e descritivo e a metodologia empregada foi a da pesquisa bibliográfica, que utilizou a análise de referências teóricas publicadas em livros, artigos científicos e documentos, para que fosse possível explicar, com 
maior aprofundamento, os assuntos contidos nesse trabalho, à luz das contribuições científicas preexistentes.

Para tanto foram analisadas várias contribuições relacionadas ao assunto em questão. Desta maneira procurou-se retirar e analisar citações de obras e autores diferentes, dos quais destacam-se Applegate, Mcfarlan \& Mckenney (1996), O’Brien (2004), Ballou (2006), Silveira, Guide \& Fernandes (2018), Ribeiro \& Gomes (2019) e Filho (2020). Sabe-se que uma boa base teórica é o alicerce para que se possa olhar os dados bibliográficos levantados e desenvolver esse estudo, indo além do que a realidade nos mostra.

\section{FUNDAMENTAÇÃO TEÓRICA}

Esta fundamentação teórica foi ordenada em cinco subtópicos, no primeiro fala-se sobre os aspectos conceituais da cadeia de suprimentos, apresentando os principais conceitos teóricos extraídos da literatura a respeito do tema. No segundo, discorreu-se sobre os conceitos de tecnologia da informação e sistemas de informação, apresentando as principais diferenças entre eles, visando uma melhor compreensão do tema. No terceiro, foram abordados a tecnologia da informação e os sistemas de informação demonstrando que eles podem ser aplicados na cadeia de suprimentos. No quarto, foi apresentado uma abordagem sobre os impactos da automatização da cadeia de suprimentos. No quinto tópico, para exemplificar esses conceitos, foi apresentado um relato de caso sobre como ocorreu a automatização dos processos em um grupo empresarial brasileiro ressaltando os resultados obtidos após a aplicação da tecnologia da informação na cadeia de suprimentos.

\subsection{Cadeia de Suprimentos}

O mercado mundial vem passando por grandes transformações, fazendo com que as empresas tenham a necessidade de se tornarem, cada vez mais, ágeis e competitivas para acompanhar esse nível de mudanças exigidas para as organizações. Isso vem mudando as relações existentes entre as empresas e seus consumidores finais, onde esses últimos estão, cada vez mais, exigentes e as empresas precisam estar mais atentas e preocupadas em atender essas necessidades. Com base nessas mudanças, é importante aprimorar a cadeia de suprimentos, visto que nesse novo cenário, as empresas entendem a necessidade de focar no desenvolvimento de todas as etapas e participantes dessa cadeia, onde deve ser constante a busca por melhores níveis de serviços com menor custo, apresentando ganhos em eficiência nos processos.

Segundo Ballou (2006) a cadeia de suprimentos é um conjunto de atividades funcionais (transporte, controle de estoques etc.) que se repetem inúmeras vezes ao longo do canal pelo qual matérias-primas vão sendo convertidas em produtos acabados aos quais se agrega valor até que tal produto chegue aos consumidores. De acordo com Bertaglia (2009, p.05), Supply Chain significa:

A cadeia de abastecimento corresponde ao conjunto de processos requeridos para obter materiais, agregar-Ihes valor de acordo com a concepção dos clientes e consumidores e disponibilizar os produtos 
para o lugar (onde) e para a data (quando) que os clientes e consumidores os desejarem.

Nesta cadeia considera-se imprescindível o pleno atendimento do consumidor, pois é o consumidor que vai, com as suas opiniões e críticas apresentar um feedback de modo que por meio destas manifestações os gestores da cadeia de suprimentos possa aprimorar suas estratégias garantindo, desta maneira, a fidelização do cliente na medida em que atende suas necessidades prementes. Chopra e Meindl (2003, p. 25) ressaltam a importância da informação para que a Supply Chain Management tenha um adequado funcionamento:

Os gerentes precisam de informações concisas para atender as necessidades dos clientes, a quantidade existente de estoque, qual o momento adequado para produzir ou embarcar produtos etc. [...], a informação é o assunto mais importante da cadeia de suprimentos, pois sem ela todos os outros aspectos não conseguiriam proporcionar um alto nível de performance. Para concretizar essa consistência das informações e dos processos de negócio, várias ferramentas foram empregadas durante os últimos anos.

Como já foi dito os gestores, praticamente, dependem de um conjunto de informações objetivas que possam revelar para eles todas as necessidades dos clientes, porque, fundamentados nestas informações, a gestão da cadeia de suprimentos, a adequada gestão de estoque e o atendimento rápido das preferências de transporte vão garantir um trabalho bem feito que vai se tornar, progressivamente, um modelo para o atendimento de situações que ainda estão por vir. $\mathrm{Na}$ opinião de Secaf (2019, p. 05)

A gestão da cadeia de produção (Supply Chain Management - SCM) é um processo amplo, complexo e que passa por constantes processos de evolução, os quais estão envolvidos na aquisição de matéria-prima, produção, armazenamento, controle de estoque, distribuição, pós-venda e alguns outros aspectos. (grifos nossos)

Não se pode esquecer da grande complexidade que caracteriza a SCM, posto que ela tem diversos pormenores que, quando não atendidos em sua plenitude, podem provocar aumento dos custos da atividade desenvolvida e comprometer o desenvolvimento de etapas cruciais dentro da cadeia de suprimentos, tais como, o transporte e a recepção dos materiais slicitados pelo cliente. Ribeiro e Gomes (2019, p. 04) destacam que:

O gerenciamento na cadeia de suprimentos demonstra uma quebra de fronteiras entre os agentes de atuação, pois antes o que era visto como rivalidade, tem seu panorama alterado para novas parcerias com potencial agregador de valor ao cliente. A abordagem não mudou, continua sendo o fluxo de materiais e a interação de processos, porém essa atuação agora é feita em conjunto e com foco estratégico, buscando a melhoria de resultados como redução de custo, desperdício e agregando valor ao consumidor final.

Ressalte-se que após o incremento dos serviços de informações integrados com a cadeia de suprimentos o que se observa, atualmente, é uma ampliação da área de atuação da SCM de modo que pode-se considerar que mais horizontes serão, ainda, expandidos posto que a principal 
característica da cadeia de suprimentos é a busca de ampliação de mercados com necessidades gerais e específicas. (Ribeiro \& Gomes, 2019). Com relação aos processos de evolução Secaf (2019) apresenta a seguinte ilustração (Figura 1):

\section{Figura 1 -}

\section{Evolução do Conceito de Cadeia de Suprimentos}

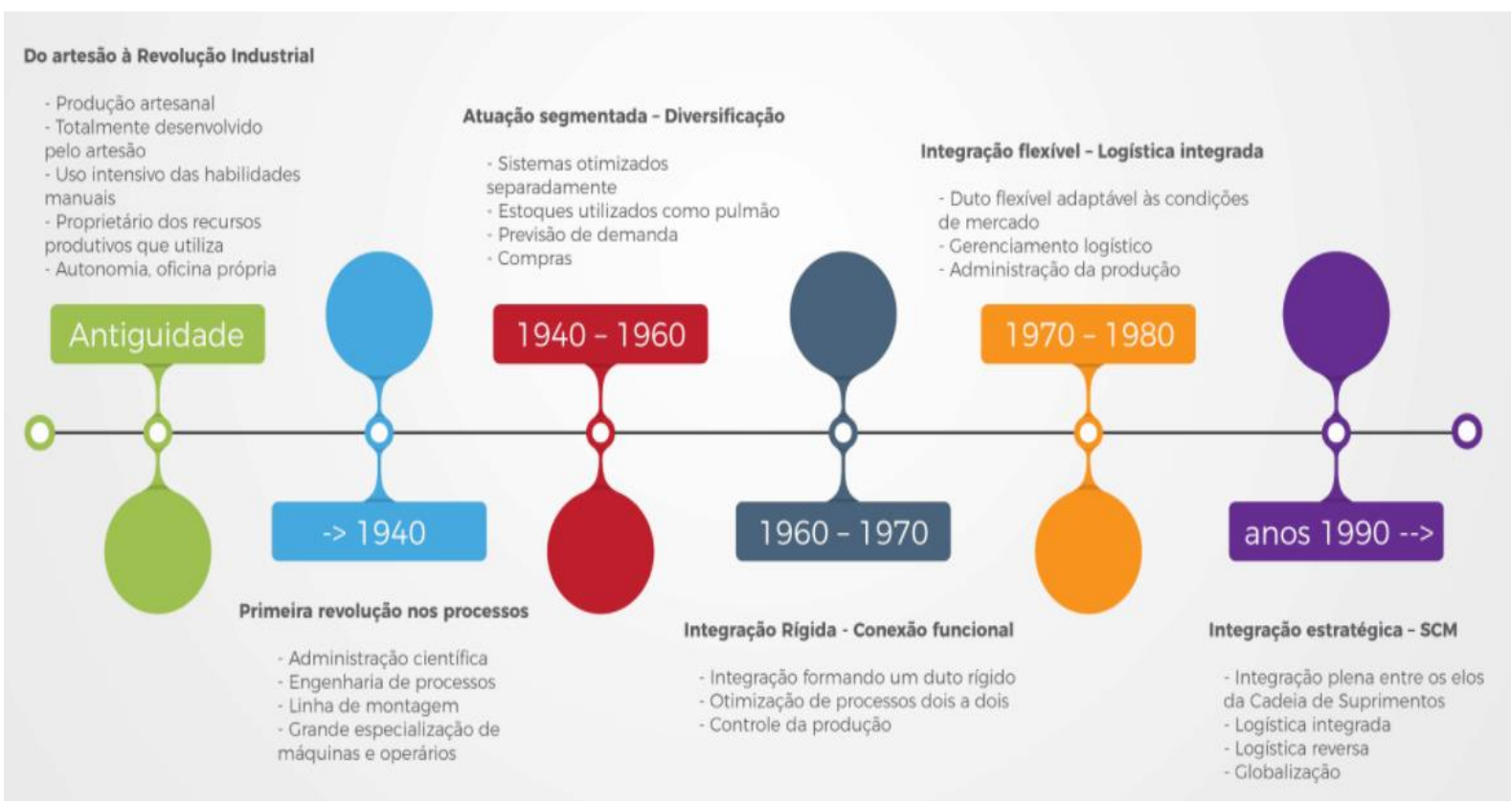

Fonte: Secaf (2019)

Felix e Gomes (2019, p. 10) afirmam "o controle das atividades relacionadas ao setor de suprimentos é uma tarefa árdua, para isso é importante o uso de ferramentas auxiliares, que promovam maior gestão do planejamento e negociação". Para que a cadeia funcione, é importante que todos os participantes do processo estejam engajados e comprometidos, onde todos estejam buscando o crescimento e aprimoramento dessa cadeia de suprimentos.

\subsection{Tecnologia da Informação e Sistemas de Informação}

A tecnologia da informação tem sido amplamente desenvolvida nas empresas, o que faz com que os administradores precisem entender seu conceito, escopo e aplicações. Devido à enorme dinâmica do campo, certos conceitos devem ser bem absorvidos como forma de criar uma base de conhecimento sólida, de forma que seja mais fácil lidar com o campo do conhecimento em rápida evolução. Esta tecnologia da informação tem produzido uma série de sistemas utilizados dentro da organização, que podem funcionar individualmente, ou de forma integrada. Embora alguns termos sejam muito semelhantes e pareçam ter o mesmo significado, é importante distingui-los, conceitualmente, para melhor compreensão quando usados. 
Para Filho (2020, p.02)

As tecnologias e os sistemas da informação proporcionam maior velocidade, expansão e acesso a um universo abrangente de informações, de maneira que se afiguram como essenciais para empresas que desejam acompanhar o mundo financeiro e os rumos da atividade que desempenha. As tecnologias e os sistemas da informação podem propiciar as informações necessárias para o sucesso da organização, além de definir as maneiras de sua apresentação, arquivo, difusão e utilização, originando novos artigos e atividades passíveis de comercialização. (Grifos nossos)

Esta situação ocorre no contexto da tecnologia da informação, sistemas de informação, e é definida da seguinte forma: Tecnologia da informação: é o componente técnico de um sistema de informação, quando se utiliza, o conhecimento científico aplicado ao processamento da informação. Sistema de informação: por conceito é o sistema pelo qual os dados são obtidos, armazenados e processados para embasamento das operações para atingir os objetivos organizacionais. (Filho, 2020)

De acordo com O'Brien (2004, p. 15), o Sistema de Informação é o "Conjunto de pessoas, procedimentos e recursos que coleta, transforma e dissemina informações em uma organização". Nesse caso, as empresas podem usar a tecnologia da informação para auxiliar nesse processo por meio de software, hardware, redes e outros componentes técnicos disponíveis. Mesmo quando se fala que Sistema de informações (SI) é considerada sinônimo, mas não é equivalente, a "Tecnologia da Informação $(\mathrm{TI})$ refere-se às tecnologias de computadores e telecomunicações que são utilizadas nas corporações, incluindo as relacionadas ao processamento e transmissão de dados e imagens". (Applegate; Mcfarlan; Mckenney, 1996). Para facilitar entendimento, neste artigo, será utilizado o termo "Tl", por ser mais comum, para representar a utilização das Tecnologias de Informação (TI), incluindo, inclusive, os sistemas de informação (SI).

\subsection{Tecnologia da Informação e Sistemas de Informação na Cadeia de Suprimentos}

Com o desenvolvimento da tecnologia da informação, as empresas enxergam a TI como um diferencial de mercado e a utilizam, estrategicamente, na integração de informações para simplificar o processo desde a produção até a disponibilização do produto ou serviço ao consumidor final. A gestão desse processo pode reduzir custos e otimizar tempo, o que é um fator importante no mercado atual. De acordo com Silveira, Guide e Fernandes, (2018, p. 04):

[...] pode-se perceber que o fluxo de informações é um elemento de grande importância nas operações logísticas. Pedidos de clientes e de ressuprimento, necessidades de estoque, movimentações nos armazéns, documentação de transporte e faturas são algumas das formas mais comuns de informações logísticas.

As tecnologias que se colocaram no mercado nos últimos anos proporcionaram também a utilização da gestão da cadeia de suprimentos, melhorando os processos logísticos e proporcionando 
meios de comunicação mais eficiente entre as empresas e possibilitando uma melhor integração de seus processos industriais e comerciais.

Para Marcadar et al. (2002, p. 25) a SCM "é um conjunto de atividades, ferramentas e software que permite a uma empresa integrar a produção, mais estreitamente, entre vários parceiros de negócio em um sistema de valor." É necessário um alinhamento entre fornecedor e consumidor final tornando a cadeia mais competitiva e com menores custos para todos, podendo ser chamado como gerenciamento da cadeia de suprimentos (Supply Chain Management). Existem atividades logísticas na cadeia de suprimentos que não podem ser esquecidas. Por muito tempo, a logística era vista apenas no contexto militar, incluindo compras, manutenção e transporte de materiais e pessoal. Ao fazer da logística uma de suas atividades, a empresa ampliou seu escopo. Segundo Fleury (1999, p. 35)

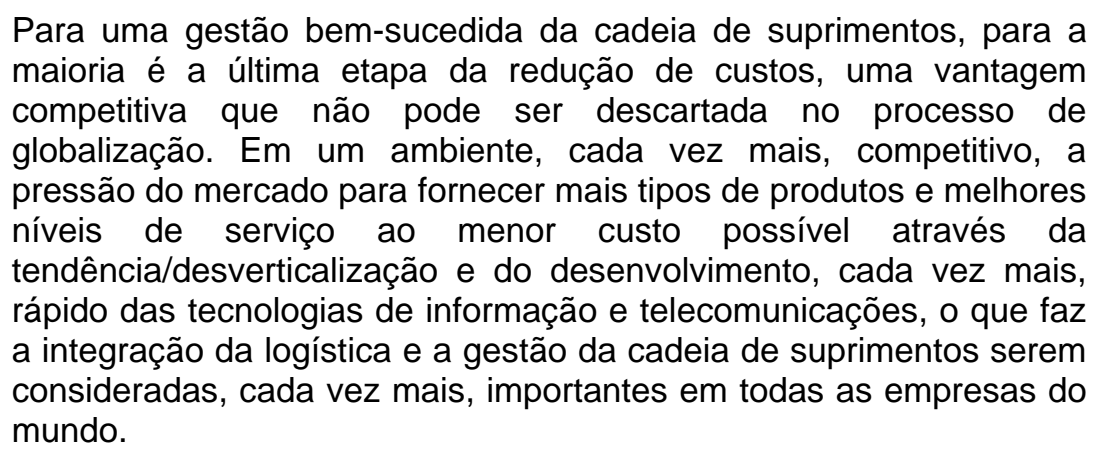

A relação de todos os envolvidos na cadeia de suprimentos começa quando o departamento comercial envia o pedido ao setor de produção. Hoje, a agilidade de entrega de produtos aos clientes depende muito dos sistemas existentes na empresa. Preparar pedidos de compra para fornecedores e suas possibilidades de entrega leva muito tempo porque envolve confecção do documento de compra, verificação de estoque, revisão das quantidades necessárias, negociação com fornecedores, respostas das solicitações de cotação, análise das respostas de cotação e aprovação de solicitação de compra. Se a corporação tiver um sistema de informação eficiente, esse tempo pode ser diminuído consideravelmente. (Ribeiro \& Gomes, 2019)

É importante ressaltar que todos os elos da cadeia de suprimentos são importantes e todos devem perceber que a agilidade é fundamental para a empresa, pois reduzirá o tempo de entrega e os custos. Todos os steps ou atividades devem ser integrados para que não haja interrupção no seu funcionamento, logo todos são responsáveis pela sua própria eficiência e pela redução do seu custo. De acordo com Chopra e Meindl (2001, p. 17)

A informação é um fator importante na tomada de boas decisões de gerenciamento da cadeia de suprimentos porque fornece conhecimento global. A tecnologia da informação fornece a capacidade de coletar essas informações e suas ferramentas de análise para tomar as melhores decisões na cadeia de abastecimento. 
Os sistemas de informações utilizados na gestão estão se tornando, cada vez mais, complexos e o escopo das informações é mais amplo para facilitar a tomada de decisões. Esses sistemas podem ser usados por compradores, fornecedores e todos que desejam controlar melhor suas atividades e trabalhar com mais eficiência.

\subsection{Impactos da Automatização da Cadeia de Suprimentos}

Tendo em vista a constante necessidade de mudança que as empresas vêm enfrentando para manter a competitividade no mercado, a tecnologia já mostrou que está sendo inserida nesse contexto para apoiar todo esse processo. Da mesma forma, o cenário tecnológico vem passando por diversas transformações e trazendo a possibilidade da integração entre todas as áreas da cadeia de suprimentos, no que diz respeito ao compartilhamento de informações, acompanhamento e melhor desempenho. Esse processo demanda, cada vez mais, inteligência estratégica e integração entre todas as etapas, exigindo integração entre todas as atividades, pois, além do menor custo, as empresas precisam buscar a maior capacidade de se relacionar com todos os parceiros da cadeia. Atualmente as tecnologias e sistemas de informações mais utilizadas nas atividades logísticas, podem ser visualizados, com as suas devidas definições, no quadro 1 , a seguir:

\section{Quadro 1 -}

Sistemas de Informações Logísticas (Tecnologias e Definições)

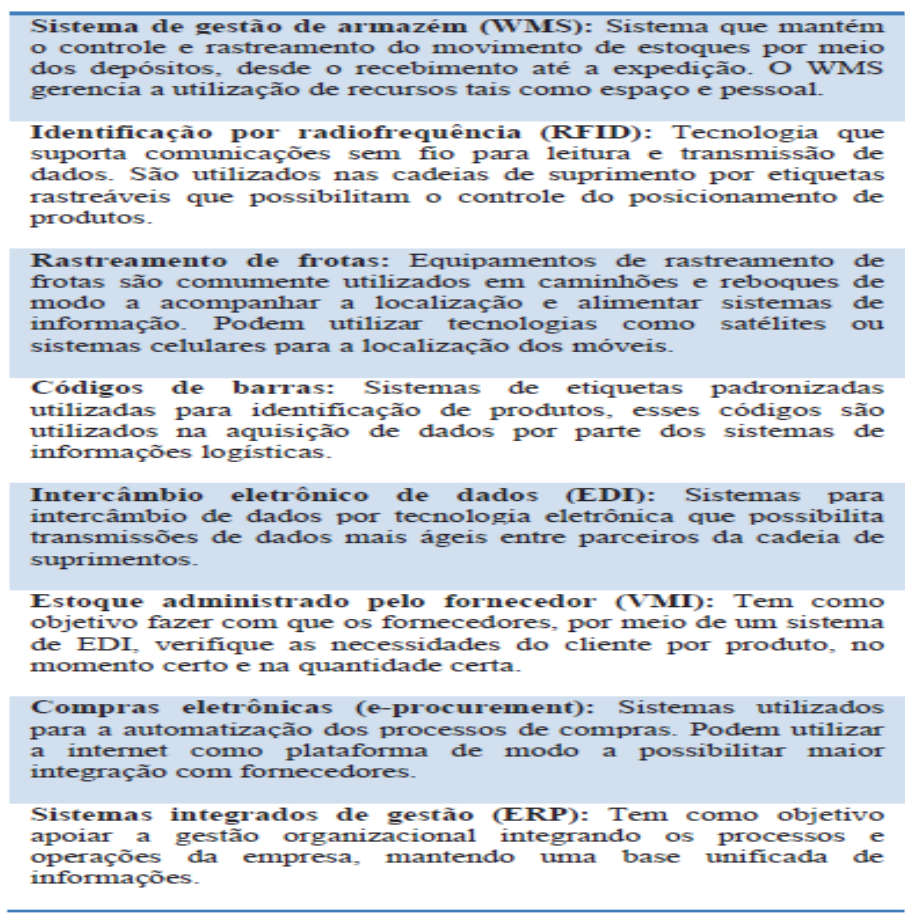

Fonte: Silveira, Guide \& Fernandes (2018)

A automação da cadeia de suprimentos possui diversas vantagens, dentre elas, apresenta-se a necessidade de uma maior visibilidade dos processos, facilitando a análise e a tomada de decisão. 
Outro ponto importante é a necessidade de automação do processo para controle de todas as etapas, com objetivo de aumentar a produtividade, reduzir perdas e excessos de estoques, favorecendo uma visão de toda a cadeia produtiva. Para fortalecer a cadeia de suprimentos, a existência de um sistema (plataforma) prepara a indústria para suportar as mudanças de mercado e responder, rapidamente, às variações na demanda, favorecendo uma análise dos dados de dentro e fora a empresa, possibilitando que os executivos reduzam o tempo gasto com atividades operacionais e possam ser mais estratégicos, visto que passam a ter um maior controle dos processos para torná-los mais eficientes. Todas essas melhorias reduzem as demandas feitas em tempo e recursos, focando mais em inovação para atender às necessidades de agilidade e adequação aos novos mercados. (Ribeiro \& Gomes, 2019)

\subsection{A Aplicação da Tecnologia da Informação na Cadeia de Suprimentos de um Grupo Empresarial Brasileiro}

Para entendimento, as empresas do grupo empresarial objeto desse estudo, formam um conglomerado de unidades fabris, unidades de envaze e vários escritórios comerciais e administrativos, distribuídos em todo território nacional, com atuação nos mais diversos segmentos de mercado, constituindo-se, dessa maneira, em um foco de interesses para várias empresas que desejam fornecer variados produtos para ela. Observando o cenário da aplicação da tecnologia na cadeia de suprimentos o grupo empresarial, objeto desse estudo, adotou um sistema ERP Integrado, - Oracle EBS, onde é possível realizar todas as etapas do processo da cadeia de Supply Chain, especialmente as relacionadas ao processo de compras (e-procurement). Nesse módulo é possível atender as demandas de compras de todas as empresas desse grupo empresarial, possibilitando que os compradores tenham um relacionamento mais próximo com os todos os fornecedores que já estão, previamente, cadastrados na ferramenta. (Silveira; Guide; Fernandes, 2018)

O uso dessa ferramenta é muito benéfico para todo o processo, pois gera flexibilidade e agilidade ao comprador no atendimento das demandas, possibilitando uma redução dos custos nas compras realizadas, resultando em economia em todas as etapas da cadeia de suprimentos e uma transparência no processo, que pode ser auditado em qualquer uma das fases. Beneficia, também, os fornecedores que passam a ser comunicados, imediatamente, sobre a demanda, pois a ferramenta possibilita uma resposta imediata às cotações, informando as suas melhores condições comerciais, gerando previsibilidade de venda para seus negócios. Essa ferramenta é de propriedade desse grupo, não havendo custo adicional para os fornecedores que participam das cotações, havendo apenas a premissa de um cadastro prévio de acordo com o segmento. (Silveira; Guide; Fernandes, 2018)

Todos os fornecedores têm a ciência que os valores apresentados na ferramenta são de sua inteira responsabilidade e que deverão conceder um desconto financeiro durante o processo de negociação, visando obter a melhor qualificação no mapa de cotações. Além do preço, os fornecedores são analisados pela qualidade e prazo de entrega. Nessa ferramenta é possível gerar as negociações por catálogo, leilões, leilões reversos e acordos de compras. Caso o fornecedor opte 
em não participar da cotação ou deseja solicitar a suspensão temporária da ferramenta para não participar das próximas negociações, pode fazê-lo a qualquer tempo.

Uma outra funcionalidade do módulo de e-procurement é a possibilidade de consulta, por parte do fornecedor, a todos os pedidos de compras gerados, bem como, a consulta das notas fiscais, trazendo mais controle dos pagamentos a receber. Dessa forma, esse sistema permite que sejam controladas todas as etapas da cadeia, como: estoque, previsão de demanda, requisição de compra, processo de cotação, negociação com fornecedores, fechamento da compra, recebimento do produto/serviço, entrada da Nota Fiscal e realização do pagamento, sendo um forte exemplo de como a tecnologia pode ser aliada no processo de organização e controle, visando a eliminação dos desperdícios, redução de custos e aumento da eficiência. (Ribeiro \& Gomes, 2019)

\section{CONSIDERAÇÕES FINAIS}

No cenário atual as empresas em processo de modernização/atualização precisam criar estratégias para manterem-se bem posicionadas no mercado. A partir da necessidade de buscar estratégias para assegurar maior qualidade, menor preço, maior agilidade e presença junto aos consumidores finais, as empresas estão encontrando esse diferencial na aplicação da tecnologia da informação aos seus processos.

Com as transformações que vêm ocorrendo, também no cenário tecnológico, a facilidade trazida pela integração das áreas da cadeia de suprimentos vem trazendo melhor desempenho para as empresas. Nesse trabalho foi possível demonstrar a importância e os impactos da aplicação da Tecnologia da Informação para o Gerenciamento da Cadeia de Suprimentos, demonstrando, de maneira razoável que, através da utilização de sistemas e de suas integrações, torna-se mais fácil a obtenção de informações para a tomada de decisões estratégicas em todas as etapas da cadeia, resultando na redução dos custos e aumento da lucratividade das empresas.

Por fim, a pesquisa revelou que, quando as empresas, do grupo empresarial estudado, conseguiram unificar os processos e assegurar a sinergia entre as áreas, ficou claro como a cadeia tornou-se mais eficiente, reforçando a importância da aplicação da tecnologia da informação. Dessa forma, concluímos que a Tecnologia da Informação é uma grande aliada das empresas nessa busca para alcançar um diferencial de mercado que possa atrair mais clientes, prestando um serviço cada vez melhor com o apoio técnico científico dos sistemas de informação.

\section{REFERÊNCIAS}

Applegate, L. M., Mcfarlan, F. W. \& Mckenney, J. L. (1996). Corporate information systems management: The issues facing senior executives (4⿳亠丷a ed.). Chicago: Irwin.

Ballou, R. H. (2006). Gerenciamento da cadeia de suprimentos: logística empresarial. Rio Grande do Sul: Bookman.

Bertaglia, P. R. (2009). Logística e gerenciamento de cadeia de abastecimento. São Paulo: Saraiva. 
Chopra, S. \& Meindl, P. (2001). Supply Chain Management: Strategy, Planning, and Operation. New Jersey: Prentice-Hall, Inc. p. 354.

Chopra, S. \& Meindl, P. (2003). Gerenciamento da cadeia de suprimentos: Estratégia, Planejamento e Operação. São Paulo: Pearson.

Felix, K. S. \& Gomes, R. L. R. (2019): Negociação e planejamento de suprimentos na construção civil. Revista Observatorio de la Economía Latinoamericana, Vol. 11. noviembre. ISSN: 16968352.

Filho, G. C. A. (2020). A gestão do conhecimento e a tecnologia da informação no melhoramento da performance da organização. Revista Científica Multidisciplinar Núcleo do Conhecimento. Ano 05, Ed. 10, Vol. 16, pp. 34-50. ISSN: 2448-0959.

Fleury, P. F. (1999). Supply Chain Management: conceitos, oportunidades e desafios de implementação. Revista Tecnologística, n. 39, fev. ISSN 1678-8532.

Marcadar, L. G., Pedron, C \& Cazella, S.O. (2002). Algum Tempo Depois... Como Grandes Empresas Avaliam o Impacto dos Sistemas ERP sobre suas Variáveis Estratégicas. Anais do 26ํㅜ Encontro da ANPAD. Salvador (BA), set.

Martins, F. C. (2011). Gestão da Cadeia de Suprimentos: Aprimoramento da Metodologia de Diagnostico e Proposição de um Método para Implantação baseado em Processos de Negócio. In: Anais do Congresso de Iniciação Científica UNIMEP, Piracicaba.

O'Brien, J. A. (2004). Sistemas de informação e as decisões gerenciais na era da Internet. $2^{\mathrm{a}}$ ed. São Paulo: Saraiva.

Ribeiro, M. A. B. \& Gomes, R. L. R. (2019). A cadeia de gestão em suprimentos: um breve estudo sobre indicadores de Acurácia. Revista Contribuciones a la Economía. Vol. 09. septiembre. ISSN: $1696-8360$.

Secaf, V. M. S. (2019). O que é cadeia de suprimentos e como promover o gerenciamento adequado para obter o sucesso nos negócios. São Paulo: Setting Consultoria. Disponível em: https://setting.com.br/blog/processos/cadeia-suprimentos/. Acesso em 14 de agosto de 2021.

Stéen, J. (2006). Improving Supply Chain Management with Advanced Planning and Scheduling: Effects and possibilities with an international perspective (Master's thesis). Linköping Institute of Technology, Linköping.

Silveira, M, Guide, R. \& Fernandes, D. (2018). A Importância dos Sistemas de Informação para Eficiência e Competitividade nas Atividades Logísticas. Memorias de la Octava Conferencia Iberoamericana de Complejidad, Informática y Cibernética (CICIC, 2018). Flórida (USA). 